Bull. Korean Math. Soc. 48 (2011), No. 4, pp. 791-812

DOI 10.4134/BKMS.2011.48.4.791

\title{
A SURVEY ON AMERICAN OPTIONS: OLD APPROACHES AND NEW TRENDS
}

\author{
Se Ryoong Ahn, Hyeong-Ohk Bae, Hyeng Keun Koo, and Kijung Lee
}

\begin{abstract}
This is a survey on American options. An American option allows its owner the privilege of early exercise, whereas a European option can be exercised only at expiration. Because of this early exercise privilege American option pricing involves an optimal stopping problem; the price of an American option is given as a free boundary value problem associated with a Black-Scholes type partial differential equation. Up until now there is no simple closed-form solution to the problem, but there have been a variety of approaches which contribute to the understanding of the properties of the price and the early exercise boundary. These approaches typically provide numerical or approximate analytic methods to find the price and the boundary. Topics included in this survey are early approaches(trees, finite difference schemes, and quasi-analytic methods), an analytic method of lines and randomization, a homotopy method, analytic approximation of early exercise boundaries, Monte Carlo methods, and relatively recent topics such as model uncertainty, backward stochastic differential equations, and real options. We also provide open problems whose answers are expected to contribute to American option pricing.
\end{abstract}

\section{Introduction}

Derivatives have dramatically revolutionized the modern world of finance; practice of corporate finance, investment, and financial services have undergone a big transformation since introduction of new derivative products. Their primary role has been hedging tools, i.e., products to be used for reducing risk associated with interest rates, foreign exchange, equity, commodity, energy, and weather. They have, however, been used also for speculative purposes, since they provide numerous ways to increase leverage. Furthermore, their prices

Received December 14, 2009.

2010 Mathematics Subject Classification. Primary 91B28; Secondary 35R35, 45G05, $65 \mathrm{C} 05$.

Key words and phrases. American option, American put, optimal exercise boundary, free boundary problem, Monte Carlo methods.

The research in the paper was supported by WCU(World Class University) program through the National Research Foundation of Korea funded by the Ministry of Education, Science and Technology (R31-2009-000-20007-0), and Ajou University Research Fellowship of 2007-2009. 
have generated signals about economic fundamentals and market conditions for the world financial community (Grossman [34]).

This spectacular success of derivative markets, however, would not have been possible, if there had been no well-accepted pricing models. A pricing model has allowed traders to base their trades on the prices discovered through it. In addition, a hedging method associated with the pricing model has induced financial institutions to make quite a large supply of derivative products by enabling them to reduce risk in their positions. A typical example of such a model is the Black-Scholes model which provides a simple closed-form formula for a European call option (or a European put option by the put-call parity) [7]. The Black-Scholes formula is a solution to a constant-coefficient parabolic partial differential equation with a terminal condition; the equation is derived based on the principle of absence of arbitrage from a set of simplifying assumptions, i.e., a geometric Brownian assumption for the underlying asset price process, no short-selling restrictions, no taxes nor transaction costs. Due to later development on risk-neutral pricing, the price can also be expressed as an expected discounted value of the option's payoff at expiration under the risk-neutral measure (see e.g., Karatzas and Shreve [50]).

Although there exists a simple pricing formula for a European option, such does not for an American option. An American option allows its holder to exercise earlier than expiration. This early exercise privilege complicates the pricing problem; the problem involves the owner's optimal choice of the time for exercise and this choice should be reflected in pricing and hedging. Under the assumption of the Black-Scholes model, the time for optimal exercise can be shown to be the first hitting time of a boundary, the early exercise boundary, in the plane consisting of pairs of the stock price and the time-to-maturity, which is to be found as a free boundary associated with the Black-Scholes partial differential equation [50]. Alternatively, the problem can be formulated as a variational inequality (see e.g., Broadie and Detemple [11]). Except for perpetual options, there is, in general, no simple formula for the early exercise boundary and option price.

Non-existence of a simple formula triggered two major trends in research on American options: In the first trend researchers seek conceptual understanding of the early exercise privilege and try to come out with a theoretical framework or an approximate formula. In the second trend they try to develop numerical methods for pricing and hedging American options, e.g., finite difference/element schemes for the free boundary value problem, or Monte Carlo methods.

In this survey we review major ideas in the first trend of research, i.e., conceptual understanding of the early exercise privilege. In our opinion the following have been prominent ideas: early attempts to obtain approximate prices $[58,9]$, the integral representation of the early exercise premium $[51,53,19]$, the analytic method of lines and its associated randomization method [17], and 
a homotopy method [80], analytic approximations of the early exercise boundary [20], and Monte Calro methods for American options. We discuss these key ideas and identify problems that need to be answered for further development of their methods. We will also discuss model uncertainty, backward stochastic differential equations, and real options.

Of course, there are other approaches which are not covered in this review. They are omitted not because they are less important but, to our view, the aforementioned methods seem best fit to our purpose.

There are already good surveys on the subject; for example, Broadie and Detemple [11] is a survey on both European and American options, Detemple [24] is a book for a comprehensive survey on American-style options, and Glasserman [32] is a book for a comprehensive treatment for Monte Calro simulation methods for American options. Our contribution is to be more up to date and cover such recent development as the homotopy method, analytic approximation to the early exercise boundary, model uncertainty, and relationship with real options and backward stochastic differential equations.

This survey is organized as follows. Section 2 sets up the American option pricing problem. Section 3 reviews early approaches to pricing of American options. Section 4 studies the integral representation of the early exercise premium and its uses. Section 5 reviews the analytic method of lines and its associated randomization method. Section 6 provides a critical review of Zhu's homotopy method [80], and Section 7 reviews analytic approximation of the early exercise boundary. Section 8 explains Monte Carlo methods, and Section 9 discusses recent topics: model uncertainty, backward stochastic differential equations, and real options. Section 10 reviews open problems and suggests directions for future research. Section 11 concludes.

\section{An American option pricing problem}

In this section we present a simple financial market model in which an American option is traded. For simplicity of exposition we focus our discussion on an American put option. There is a put-call symmetry between American put and American call options [33, 56, 69, 15, 16] and the approaches we review in this survey can be applied equally to an American call option with continuous dividends with modification of parameters. ${ }^{1}$

There are a stock and a bond in the financial market. The bond is risk-free and assumed to have a constant rate of return $r$. The price $S(t)$ of the stock

\footnotetext{
${ }^{1}$ In a geometric Brownian motion setting assumed in this paper the put-call symmetry is stated as follows:

$$
C(S, K, r, \delta, T)=P(K, S . \delta, r, T),
$$

where $C, P$ denote prices of American call and put options, respectively, and the other notation is the same as in the rest of the paper.
} 
evolves according to the following equation

$$
\frac{d S(t)}{S(t)}=\mu d t+\sigma d \tilde{B}(t), S(0)=S_{0},
$$

where $\mu$ and $\sigma$ are constants describing the mean and volatility of the stock return, and $\tilde{B}(t)$ is a standard Brownian motion on a filtered probability space $\left(\Omega, \mathcal{F},\left\{\mathcal{F}_{t}\right\}_{t \geq 0}, P\right)$. The stock pays dividends at a constant rate $\delta$, namely, the amount of dividend paid over the infinitesimal time interval $[t, t+d t)$ is equal to $\delta S(t) d t$.

We assume that there are no taxes, no transaction costs and no short-sale constraints. Portfolio strategies, particularly self-financing strategies starting with a given initial wealth, are defined as in a standard financial model (see e.g., Chapter 1, [50]).

By Girsanov' theorem there exists an equivalent martingale measure $Q$ under which the stock price evolves as follows

$$
\frac{d S(t)}{S(t)}=(r-\delta) d t+\sigma d B(t), S(0)=S_{0},
$$

where $B(t) \equiv \tilde{B}(t)+\frac{\mu-r}{\sigma} t$ is a Brownian motion under $Q$.

We now consider an American put option written on the stock with a strike price $K$ and maturity $T<\infty$. The option pays $g(S(\tau)) \equiv(K-S(\tau))^{+}$to the holder when it is exercised at $\tau$. Suppose $S(t)=S$, then we define

$$
P(T-t, S) \equiv \operatorname{ess} \sup _{t \leq \tau \leq T} E^{Q}\left[e^{-r(\tau-t)} g(S(\tau)) \mid \mathcal{F}_{t}\right]
$$

where $\tau$ runs all $\left\{\mathcal{F}_{s}\right\}_{t \leq s \leq T}$-stopping times. Bensoussan [6] and Karatzas [49] have shown that $P(T-t, S)$ is a unique fair price of the American put at time $t$ and the price at time 0 is equal to the smallest initial wealth required such that there exists a self financing strategy whose wealth process $X(u)$ satisfies

(i) $X(u) \geq g(S(u))$ for all $0 \leq u<T$,

(ii) $X(T)=g(S(T))$.

\section{The free boundary value problem}

We denote the American option price in (2) by $f(u, S):=P(u, S)$. The variable $u:=T-t$ stands for time until expiration of the option. Mckean [58] and Van Moerbeke [76] have shown that the fair value of the American put option is a solution to the following free boundary value problem ${ }^{2}$.

$$
\mathcal{L} f(u, S) \equiv \frac{\sigma^{2}}{2} S^{2} f_{S S}+(r-\delta) S f_{S}-r f-f_{u}=0 \text { for } S>\underline{S}
$$

subject to the following boundary conditions

$$
\lim _{S \uparrow \infty} \max _{0 \leq u \leq T} f(u, S)=\lim _{S \uparrow \infty} g(S)=0,
$$

\footnotetext{
${ }^{2}$ Here subscripts denote partial derivatives, i.e., $f_{S S}=\frac{\partial^{2} f}{\partial S^{2}}, f_{S}=\frac{\partial f}{\partial S}$ and $f_{u}=\frac{\partial f}{\partial u}$.
} 


$$
\lim _{S \downarrow \underline{S}(u)} f(u, S)=g(\underline{S}(u)), \lim _{S \downarrow \underline{S}(u)} f_{S}(u, S)=\frac{\partial g(S)}{\partial S}=-1
$$

and terminal conditions

$$
\begin{gathered}
\underline{S}(0+)= \begin{cases}K & \text { if } r \geq \delta, \\
\frac{r K}{\delta} & \text { if } r<\delta,\end{cases} \\
f(0, S)=g(S(T)) \text { for } S>0 .
\end{gathered}
$$

Furthermore,

$$
f(u, S)=g(S) \text { for } S \leq \underline{S}(u), u \in[0, T],
$$

and

$$
f(u, S) \geq g(S) \text { for } u \in[0, T], S>0 .
$$

Here, $\underline{S}$ is called the early exercise boundary and the optimal exercise time is the first hitting time of the stock price at the boundary.

Equivalently, the function $f(u, S)$ can be shown to be a solution to the following linear complementarity problem (LCP) [24]:

$$
\begin{gathered}
\mathcal{L} f \cdot(f-g)=0, \\
f(u, S) \geq g(S), \quad \mathcal{L} f \leq 0
\end{gathered}
$$

subject to the boundary conditions

$$
\begin{aligned}
\lim _{S \uparrow \infty} \max _{0 \leq u \leq T} f(u, S) & =\lim _{S \uparrow \infty} g(S)=0, \\
\lim _{S \downarrow 0} \max _{0 \leq u \leq T} f(u, S) & =\lim _{S \downarrow 0} g(S)=K, \\
f(0, S) & =g(S(T)) .
\end{aligned}
$$

In the LCP formulation the early exercise boundary is not shown explicitly, but rather should be discovered as a part of a solution to the problem. It is well-known that the LCP formulation is equivalent to variational inequality [11].

\section{Early approaches and ideas}

\subsection{A perpetual American option}

McKean, Samuelson, and Merton [58, 68, 59] derived an explicit analytic solution to the free boundary value problem for the case $T=\infty$, i.e., for a perpetual American option. In this case the derivative with respect to time, $f_{u}$, disappears in the equation (3), because time is homogeneous in an infinite horizon problem. That is, the equation (3) becomes a linear homogeneous second-order ordinary differential equation, which has a known general solution. By the boundary and terminal conditions, one can derive the following formula for an American put:

$$
P(S)= \begin{cases}(K-\underline{S})\left(S \underline{S}^{-1}\right)^{\gamma} & \text { when } S \geq \underline{S}, \\ K-S & \text { when } 0<S<\underline{S},\end{cases}
$$


where

$$
\underline{S}=\frac{\gamma K}{\gamma-1}, \quad \gamma:=-\frac{1}{\sigma}\left[\nu+\sqrt{\nu^{2}+2 r}\right] \text { for } \nu=\frac{r-\delta}{\sigma}-\frac{1}{2} \sigma,
$$

see $[50]$.

\subsection{Trees and finite difference schemes}

Soon after the discovery of the simple formula for a perpetual American option price, people noticed genuine difficulty of finding an analytic solution to the free boundary value problem or the LCP. Instead, they started to apply known numerical techniques such as lattices and finite difference methods.

Lattices, also known as trees, are (weak) discrete approximations to a continuous-time process. Parkinson was the first to use a trinomial lattice to price an American option [63]. Cox, Ross, and Rubinstein [22] and Rendleman and Bartter [65] applied binomial trees to price both European and American options. Kim and Byun [52] studied properties of the optimal exercise boundary in a binomial tree. For further development of the lattice methods, see Broadie and Detemple [11].

Brennan and Schwartz [8] and Schwartz [70] were the first to use finite difference methods to the LCP problem in order to price American options. Brennan and Schwartz, in particular, showed the equivalence of the explicit finite difference scheme and a trinomial tree and also established the equivalence of the implicit finite difference scheme and an infinite lattice.

The trees and the finite difference schemes are now treated as standard numerical procedures to price American options in popular textbooks such as Hull's book [39].

\subsection{Analytic approximations}

There were also proposals for approximate analytic solutions. Johnson [47] used the observation that an American put on a non-dividend paying stock is never exercised earlier than expiration if the strike price is increasing over time at the risk-free rate, and thus, is equivalent to a European put. Therefore, the American put price has both the lower bound and the upper bound; the lower bound is the price of a European put with the same terms as the American put and the upper bound is that of a European put with a strike price adjusted upward to $K e^{r T}$. Johnson derived an approximate formula which is a weighted average of the lower and upper bounds. His approximation, however, was totally ad hoc and lacked any justification for convergence to the true price.

Geske and Johnson [30] derived an approximation assuming that the American option can be exercised only at discrete times. In this case the option at a current time can be viewed as an option on an option at later periods, i.e., a compound option. They applied a pricing formula similar to that of a compound option to get an approximation to the American option price. They claimed that the approximation converges to the true value as the number of 
periods increases to infinity, however, no proof was given in their paper. The approximation involves distribution functions of $n$-variate normal distributions with $n$ early exercise periods, and becomes impractical as $n$ becomes large. They proposed Richardson extrapolation as a method to expedite convergence.

MacMillan [57] and Barone-Adesi and Whaley [5] proposed a quadratic approximation by considering the equation for the early exercise premium, $\varepsilon(T, S):=P(T, S)-p(T, S)$, where $p(T, S)$ is the price of a European put with the same terms as the American put: Since both $P(T, S)$ and $p(T, S)$ satisfy the equation (3) in the region above the early exercise boundary, $\varepsilon(T, S)$ also satisfies the equation in the region. Normalizing $K=1$ and writing $\varepsilon(T, S)=K(T) h(K(T), S)$ where $K(T)=1-e^{-r T}$, the equation (3) becomes

$$
\frac{\sigma^{2}}{2} S^{2} h_{S S}+(r-\delta) S h_{S}-\frac{r}{K(T)} h-r(1-K(T)) h_{K}=0 .
$$

By assuming $(1-K(T)) h_{K}=0$, the above equation can be approximated by the second-order ordinary differential equation, which is called the quadratic approximation:

$$
\frac{\sigma^{2}}{2} S^{2} h_{S S}+(r-\delta) S h_{S}-\frac{r}{K(T)} h=0
$$

For a given $T$, an analytic solution to the free boundary value problem with the quadratic approximation for the early exercise premium can be obtained in a way similar to that of the perpetual option. The resulting approximate formula is expressed as the sum of the European option price and an approximation of the early exercise premium, while the latter resembling the formula for the price of a perpetual put option. Barone-Adesi and Whaley claimed that the approximation is good for an option with maturity less than or equal to one year, but the error tends to be large for longer term options.

\section{An integral representation of the early exercise premium}

An essential difference between an American option and its European counterpart is its early exercise privilege. The market price of this privilege is called the early exercise premium and equal to the difference between the American option price and the European option price.

As discussed in the previous section, MacMillan, Barone-Adesi and Whalley approximated the equation for the early exercise premium by a second-order ordinary differential equation and derived an approximate analytic formula for it. There occurred a conceptual and methodological break-through, however, when a few researchers discovered a rigorous integral representation of this early exercise premium (Kim [51], Jacka [45], and Carr, Jarrow, and Myneni [19]). The following theorem provides the representation:

Theorem 4.1. Let $p(T, S)$ be the fair price of a European option with strike price equal to $K$, time to maturity equal to $T$ when the current stock price is 
S. Then,

$$
P(T, S)=p(T, S)+\int_{0}^{T} e^{-r(T-t)}(r K-\delta S(T-t)) \chi_{\{S(T-t)<\underline{S}(T-t)\}} d t,
$$

where $\chi_{A}$ denotes its characteristic function, i.e., $\chi_{A}(x)=1$ if $x \in A$ and $\chi_{A}(x)=0$ if $x \in \Omega-A$.

The representation provides an economic interpretation of the early exercise premium: it is equal to the sum (or integral) of the present discounted values of the difference between interest $r K$ from the revenue $K$ and the dividend $\delta S$ foregone from the sale of a share of the stock, when the option is early exercised.

The representation also provides a functional equation for the early exercise boundary; since $P(T, S)=K-\underline{S}(T)$ at the boundary,

(8) $K-\underline{S}(T)=p(T, \underline{S}(T))+\int_{0}^{T} e^{-r(T-t)}(r K-\delta S(T-t)) \chi_{\{S(T-t)<\underline{S}(T-t)\}} d t$,

And this equation can be used to compute the early exercise boundary numerically. See [51] and [53] for numerical methods which try to solve the functional equation (8) iteratively.

The representation in the equation (7) and the functional equation (8) are applied in a number of ways in the literature: Huang, Subrahmanyam, and Yu [38] have employed step functions, and $\mathrm{Ju}$ [48] a piecewise exponential function to approximate the early exercise boundary and used the equations to study pricing and hedging of American options. Gao, Huang, and Subrahmanyam [29] have applied the representation and the functional equation for pricing and hedging of American Barrier options. Broadie and Detemple [10] have used the representation to obtain an upper bound for the price of an American option.

\section{An analytic method of lines and randomization of the expiration date}

The method of lines refers to approximating a parabolic partial differential equation with a space variable and a time variable by using a numerical derivative with respect to one variable [60]. The equation is then approximated by a series of ordinary differential equations. Applied to our American put problem by using a numerical derivative with respect to time, the approximation becomes

$$
\frac{\sigma^{2}}{2} S^{2} f_{S S}\left(u_{n}, S\right)+(r-\delta) S f_{S}\left(u_{n}, S\right)-r f\left(u_{n}, S\right)+\frac{f\left(u_{n}, S\right)-f\left(u_{n-1}, S\right)}{\Delta_{N}}=0,
$$

where $0=u_{0}, u_{1}, \ldots, u_{N}=T$ is an equal partition of $[0, T]$ and $\Delta_{N}=u_{n}-$ $u_{n-1}=\frac{T}{N}$. Then, an approximation of the option price and the early exercise boundary can be found by solving the ordinary differential equation successively 
with the boundary conditions. By proceeding this way Carr and Faguet [18] have obtained analytic approximation to the American put price.

This method has interesting economic interpretations: As explained in [18], the American option can be regarded as a perpetual option, but time decay can be modeled as credit downgrades occurring according to a random jump of a Poisson process. Namely, there are $N$ credit classes of the option and it experiences a downgrade to the next credit class at the next jump time of a Poisson process with intensity $\lambda_{N}=\frac{1}{\Delta}=\frac{N}{T}$. Alternatively, the option can be regarded as maturing at the $N$-th jump $\tau_{N}$ of a Poisson process with the same intensity, which is an interpretation by Carr [17]. According to these interpretations, the method of lines is a way to approximate the price of a finite maturity American option by a series of perpetual options with random maturities.

The random maturity time $\tau_{N}$ has expectation and variance

$$
\begin{aligned}
E\left[\tau_{N}\right] & =\frac{T}{N}+\cdots+\frac{T}{N}=T \\
\operatorname{Var}\left(\tau_{N}\right) & =\frac{T^{2}}{N^{2}}+\cdots+\frac{T^{2}}{N^{2}}=\frac{T^{2}}{N} .
\end{aligned}
$$

Therefore,

$$
\lim _{N \rightarrow \infty} \operatorname{Var}\left(\tau_{N}\right)=0
$$

That is, $\tau_{N}$ converges to $T$ in probability as $N \rightarrow \infty$. This fact provides justification for approximation. Carr and Faguet, however, have not given a proof of convergence of the prices with random maturities to the true option price with a fixed maturity as $N$ tends to infinity. Nevertheless, their approximation seems good in computational point of view. As a demonstration, we present an application of their approximation to the behavior of the exercise boundary near expiration.

For demonstration we assume $\delta=0$ and let $\hat{b}_{2}\left(\frac{T}{N}\right):=\underline{S}_{N, 1}$ denote the free boundary decided after the first arrival among $N$ arrivals for a given $N$. Intuitively, this approximates the early exercise boundary and we expect that the sequence $\left\{\hat{b}_{2}\left(\frac{T}{N}\right): N=1,2 \ldots,\right\}$ generates delicate information on the actual boundary near expiration. Carr and Faguet's formula reads

$$
\hat{b}_{2}\left(\frac{T}{N}\right)=K\left(\frac{\left(\varepsilon_{N}-\frac{2 r}{\sigma^{2}}\right) r T}{N+r+r\left(\varepsilon_{N}-\frac{2 r}{\sigma^{2}}\right)}\right)^{\frac{2 r}{\sigma^{2}+\varepsilon_{N}}},
$$

where $\varepsilon_{N}$ is given by

$$
\varepsilon_{N}=\sqrt{\frac{1}{4}\left(1+\frac{2 r}{\sigma^{2}}\right)^{2}+\frac{2 N}{\sigma^{2}}} .
$$


In the following simulation the lower curve is $\hat{b}_{2}$ and it is evaluated and interpolated on a nonuniform partition $\pi_{1000}$ of $[0, T]$,

$$
\pi_{1000}:=\left\{\frac{T}{1000}, \frac{T}{999}, \ldots, \frac{T}{2}, T\right\}
$$

with $T=0.01$ which is approximately equal to 4 days. We remark that the partition points are clustered at time 0, meaning that all the information is concentrated near expiration.

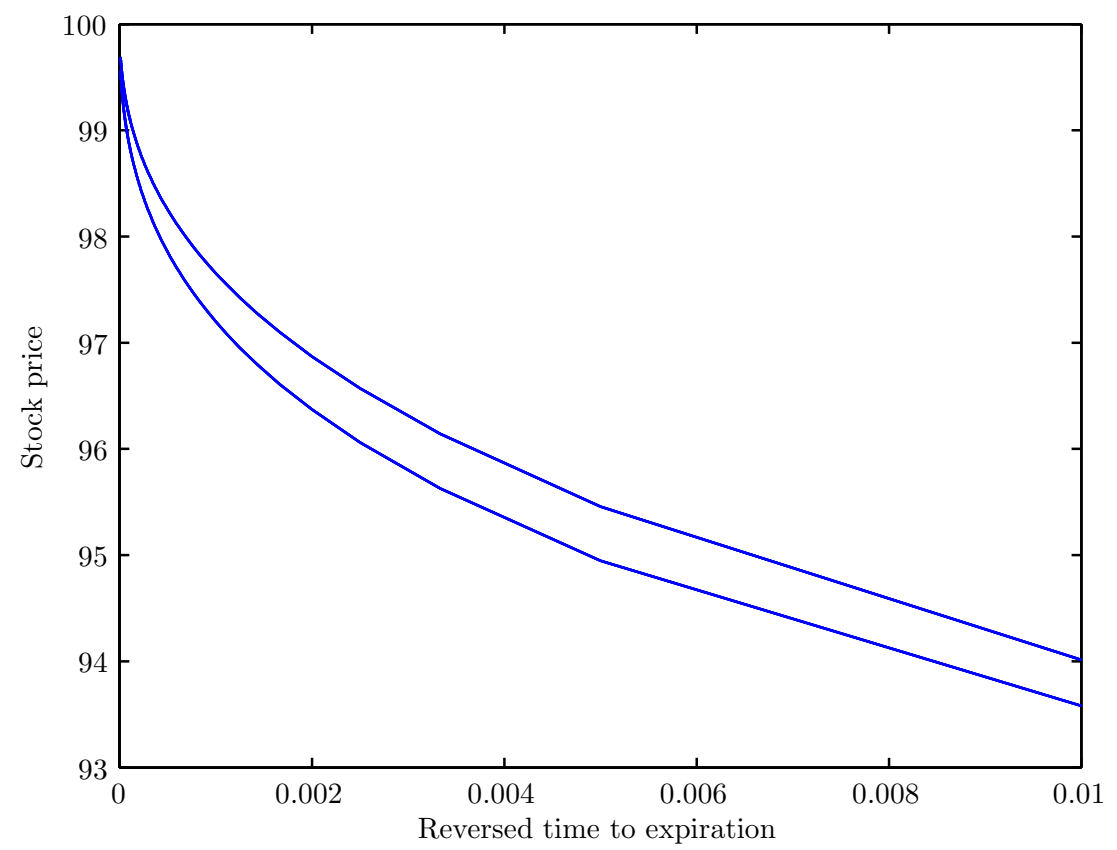

Figure 1. Comparison between two approximations to the early exercise boundary with $K=100$, interest rate $=0.1$, volatility $=0.3$, time unit $=$ year

The upper curve serves as a benchmark and gives the value of the following function on $\pi_{1000}$ :

$$
\hat{b}_{1}(t)=K \exp \left[-\sqrt{\sigma^{2} t \log \left(v^{2}(t) C t\right)}\right],
$$

where $C=\frac{8 \pi r^{2}}{\sigma^{2}}$ and $v(t)=1+\frac{1}{\log (C t)}$. The function $\hat{b}_{1}$ in (9) is claimed to be a sharp approximation to the free boundary near expiration (see [62]). As we see, the difference between $\hat{b}_{1}$ and $\hat{b}_{2}$ seems bounded by $\hat{b}_{1}(T)-\hat{b}_{2}(T)$ which goes to zero as $T$ gets smaller. Hence, the lower curve generated by Carr and Faguet's formula can be regarded as a reasonably good approximation to the 
free boundary near expiration. Carr and Faguet have shown that their method can generate good approximations also for options away from expiration by using the Richardson extrapolation. Jang and Koo [46] have also shown that the randomization method can be applied to the case where the volatility is time-varying in accordance with a two-state regime switching process.

\section{A homotopy method}

Another approach to find the price of an American put is a homotopyanalysis method proposed by Zhu [80].

Zhu has constructed a homotopy in which there is a deformation of boundary value problems parameterized by $p$ where $p$ ranges from 0 to 1 ; for $p=0$ the boundary value problem has an easy solution and for $p=1$ the boundary value problem provides the American option price and the early exercise boundary. He has, then, assumed the homotopy's analyticity and taken a Taylor series expansion around $p=0$. By plugging in $p=1$ to the series, he has obtained the American put price together with the early exercise boundary.

We now proceed to explain Zhu's idea in more detail. For simplicity, we assume that the stock pays no dividend and the strike price is equal to 1 . By introducing $\gamma:=\frac{2 r}{\sigma^{2}}$, the relative interest rate to the volatility of the underlying asset price, one gets the following version of free boundary value problem:

$$
\begin{aligned}
f_{u}-S^{2} f_{S S}-\gamma S f_{S}+\gamma f & =0, \\
f(0, S) & =\max (1-S, 0), \\
f(u, \underline{S}(u)) & =1-\underline{S}(u), \\
f_{S}(u, \underline{S}(u)) & =-1, \\
\lim _{S \uparrow \infty} f(u, S) & =0,
\end{aligned}
$$

where $\underline{S}(u)$ is the optimal exercise boundary. With intention of flattening boundary Zhu uses the change of variable ${ }^{3}$

$$
x=\ln \frac{S}{\underline{S}(u)}
$$

to get

$$
\begin{aligned}
f_{u}-f_{x x}-(\gamma-1) f_{x}+\gamma f & =\frac{\underline{S}}{\underline{S}(u)} f_{x}, \\
f(0, x) & =0, \\
f(u, 0) & =1-\underline{S}(u), \\
f_{x}(u, 0) & =-\underline{S}(u), \\
\lim _{x \uparrow \infty} f(u, x) & =0 .
\end{aligned}
$$

\footnotetext{
${ }^{3}$ The change of variable was previously done by Wu and Kwok [78].
} 
Zhu constructs two unknown functions $\bar{f}(u, x, p)$ and $\bar{B}(u, p)$ with parameter $p \in[0,1]$ that satisfy

$$
\begin{aligned}
(1-p) \mathcal{L}\left[\bar{f}(u, x, p)-\bar{f}_{0}(u, x)\right] & =-p\{\mathcal{A}[\bar{f}(u, x, p), \bar{B}(u, p)]\}, \\
\bar{f}(0, x, p) & =(1-p) \bar{f}_{0}(0, x), \\
\bar{f}(u, 0, p) & =1-\bar{B}(u, p), \\
\bar{f}_{x}(u, 0, p)+\bar{B}(u, p) & =(1-p)\left[1+\frac{\partial \bar{f}_{0}}{\partial x}(u, 0)-\bar{f}_{0}(u, 0)\right], \\
\lim _{x \uparrow \infty} \bar{f}(u, x, p) & =0,
\end{aligned}
$$

where $\mathcal{L}$ is a differential operator defined as follows:

$$
\mathcal{L}=\frac{\partial}{\partial u}-\frac{\partial^{2}}{\partial x^{2}}-(\gamma-1) \frac{\partial}{\partial x}+\gamma
$$

and $\mathcal{A}$ is a functional defined by the equation

$$
\mathcal{A}[\bar{f}(u, x, p), \bar{B}(u, p)]=\mathcal{L}(\bar{f})-\frac{\underline{S}_{u}(u, p)}{\underline{S}(u, p)} f_{x}(u, x, p) .
$$

Notice that, when $p=0,(12)$ is solvable and the case $p=1$ is (11), what we want to solve. To attack this question, Zhu expresses the functions $\bar{f}(u, x, p)$ and $\bar{B}(u, p)$ as a Taylor series expansion around $p=0$

$$
\bar{f}(u, x, p)=\sum_{m=0}^{\infty} \frac{\bar{f}_{m}(u, x)}{m !} p^{m}, \quad \bar{B}(u, p)=\sum_{m=0}^{\infty} \frac{\bar{B}_{m}(u)}{m !} p^{m},
$$

where

$$
\bar{f}_{m}(u, x)=\left.\frac{\partial^{m}}{\partial p^{m}} \bar{f}(u, x, p)\right|_{p=0}, \quad \bar{B}_{m}(u)=\left.\frac{\partial^{m}}{\partial p^{m}} \bar{B}(u, p)\right|_{p=0} .
$$

It turns out that the coefficients in the series are solutions to auxiliary equations which are solvable.

However, what is missing here is theoretical evidence of convergence of the series. To show convergence, we must be able to show that

$$
\lim _{m \rightarrow \infty} \frac{m}{m+1}\left|\frac{\bar{f}_{m+1}}{\bar{f}_{m}}\right| p<1
$$

for $p \in[0,1]$. But, Zhu has left the convergence as a conjecture and provided only numerical evidence. Moreover, one has to find $\bar{f}_{n}(u, x)$ and $\bar{B}_{n}(u)$ recursively using $\bar{f}_{n-1}(u, x)$ and $\bar{B}_{n-1}(u)$ and it requires a recursive procedure of solving differential equations to arrive at (13). Therefore, (13) with $p=1$ is not a closed-form in an ordinary sense. 


\section{Analytic approximation to the early exercise boundary and the tail of the boundary}

Under the current situation in which we don't have any exact closed-form expression for the exercise boundaries of American put options, information on parts of an exercise boundary are valuable. Some detailed information of the boundaries near expiration and boundaries far from expiration are available.

\subsection{The early exercise boundary near expiration}

There has been research which tries to understand behavior of the early exercise boundary near expiration. For instance, there have been approximations proposed by Kuske and Keller [54], Bunch and Johnson [14], and Stamicar, Sevcovic and Chadam [71] for options near maturity. These approximations are summarized succinctly in Chen and Chadam [20]:

$$
\begin{aligned}
(K K) & 9 \pi \gamma^{2} t \alpha^{2}(t) e^{2 \alpha(t)} & \sim 1, \\
(B J) & 4 \gamma^{2} t \alpha(t) e^{2 \alpha(t)} & \sim 1-\frac{\gamma^{2}}{2(1+\gamma)^{2}}, \\
(S S C) & 4 \pi \gamma^{2} t e^{2 \alpha(t)} & \sim 1,
\end{aligned}
$$

where $\alpha(t):=\frac{s^{2}(t)}{4 t}, s(t)$ is the re-scaled exercise boundary with $t$ being time until expiration (see $(P)$ below). Recently, Chen and Chadam [20] have confirmed with mathematical rigor that $(S S C)$ is correct; they have derived and proved high-order asymptotic expansions for the early exercise boundary near expiration.

Assuming that the asset price $S$ satisfies (1) with no dividends paid, $\delta=0$, they start from the following free boundary problem which is essentially the same as (10):

$$
(F) \begin{cases}f_{u}-\frac{1}{2} \sigma^{2} S^{2} f_{S S}-r S f_{S}+r f=0 & u>0, \underline{S}(u)<S, \\ f(u, S)=K-S, f_{S}(u, S)=-1 & u>0,0<S \leq \underline{S}(u), \\ \lim _{S \uparrow \infty} f(u, S)=0 & u>0,0<S, \\ \underline{S}(0)=K & u=0, \\ f(0, S)=\max (K-S, 0) & u=0,0<S .\end{cases}
$$

By change of variables, $u=\frac{\sigma^{2}}{2} t, S=K e^{x}$, and setting

$$
p(t, x):=\frac{1}{K} P(u, S), \underline{s}(t):=\ln \frac{\underline{S}(u)}{K}, \gamma=\frac{2 r}{\sigma^{2}},
$$

one obtains

$$
(P) \begin{cases}p_{t}-p_{x x}-(\gamma-1) p_{x}+\gamma p=0 & t>0, \underline{s}(t)<t \\ p(t, x)=1-e^{x}, p_{x}(t, x)=-e^{x} & t>0,0<t \leq \underline{s}(t), \\ \lim _{x \uparrow \infty} p(t, x)=0 & t>0, x \in \mathbb{R} \\ \underline{s}(0)=0 & t=0, \\ p(0, x)=\max \left(1-e^{x}, 0\right) & t=0, x \in \mathbb{R} .\end{cases}
$$


Chen and Chadam [20] analyze $\underline{s}(t)$ near $t=0$ by looking at asymptotic expansion for $\alpha(t):=\frac{\underline{s}^{2}(t)}{4 t}$ so that they get a sharp estimate for the early exercise boundary. Their result is

$$
4 \pi \gamma^{2} t e^{2 \alpha(t)} \sim 1
$$

for each sufficiently small positive $t$. In other words, one derives

$$
\alpha(t) \sim-\xi(t)
$$

where $\xi(t):=\log \sqrt{4 \pi \gamma^{2} t}$. From this we get the following approximations to $\underline{s}(t)$ and $\underline{S}(u)$ :

$$
\underline{s}(t) \sim-\sqrt{-2 t \log \left(4 \pi \gamma^{2} t\right)}, \quad \underline{S}(u) \sim K \exp \left[-\sqrt{-\frac{4 u}{\sigma^{2}} \log \left(\frac{8 \pi \gamma^{2}}{\sigma^{2}} u\right)}\right]
$$

for small $t$ and $u$. In fact, they have the following asymptotic expansion for $\alpha$ :

$$
\begin{aligned}
& \alpha(t)=-\xi(t)-\frac{1}{2 \xi(t)}+\frac{1}{8 \xi^{2}(t)}+\frac{17}{24 \xi^{3}(t)}-\frac{51}{64 \xi^{4}(t)}-\frac{287}{120 \xi^{5}(t)}+\frac{199}{32 \xi^{6}(t)} \\
&(14) \quad+O\left(\xi^{-7}(t)\right) .
\end{aligned}
$$

To reach (14), first, they use Green's representation for solutions of the linear parabolic PDE in $(P)$. Next, one isolates an equation for the free boundary $\underline{s}(\cdot)$. Finally, they derive an equation for $\alpha(\cdot)$ and analyze it to obtain (14).

\subsection{The early exercise boundary far away from expiration}

On the other hand, one can also obtain the behavior of the free boundary for a time far away from expiration. This information sheds insight to the shape of the boundary by providing knowledge about how fast the boundary becomes flat. Ahn, Choe, and Lee [1] have provided an estimate for the speed.

Suppose that $\delta=0$. For any given $\gamma=\frac{2 r}{\sigma^{2}}>0$ it holds that

$$
0<\underline{s}(t)-s_{*} \leq N_{1} e^{-\frac{(\gamma+1)^{2}}{4} t}, \quad t \geq 0
$$

where $s_{*}$ is the bounded limit of $\underline{s}(\cdot)$ and constant $N_{1}$ depends only on $\gamma$.

In this case one needs an integral equation for $\underline{s}(\cdot)$ :

$$
G(t, \underline{s}(t))=-\gamma \int_{0}^{t} G(t-s, \underline{s}(t)-\underline{s}(s)) \underline{s}^{\prime}(s) d s,
$$

where $G$ is the fundamental solution corresponding to the second order parabolic partial differential equation in $(P)$, that is,

$$
G(s, y)=\frac{1}{\sqrt{4 \pi s}} \exp \left\{-\frac{(y+(\gamma-1) s)^{2}}{4 s}-\gamma s\right\}, \quad s>0, y \in \mathbb{R} .
$$

Using (16) and the convexity of $\underline{s}(\cdot)$, one can prove (15). 


\section{Monte Carlo methods}

Monte Carlo simulation has been the most popular method for pricing complex derivatives since its introduction by Boyle [13]. In particular, when pricing involves multiple risk sources, the resulting mathematical problem is multidimensional and traditional numerical methods such as trees or finite difference schemes are not practical, the only practical method available is usually Monte Carlo simulation.

This popular method, however, had a serious problem for American option pricing because of its inherent forward nature. When pricing a derivative, the method starts by simulating many paths of prices under the risk neutral probability measure. Then cash flows of the derivative for each simulated path are computed and discounted to get their total present value. Then, the average of all the present values on the simulation paths is the derivative's current price. When trying to apply the method to an American option, people discovered that it was not easy to decide the optimal exercise time of the option on each path, since it was not easy to know the continuation value of the option at a point of time on the path. In this section we review approaches to get over the difficulty and use Monte Carlo simulation to price American options. For a more comprehensive review on this topic, see Glasserman [32].

The first class of approaches to extend Monte-Carlo pricing to Americanstyle options is approximating the early exercise boundary based on average future values. Tilley [73] has proposed the following method: At a point of time all the paths are reordered according to the price levels and grouped into a number of bundles, and the continuation value is computed as the average present value of future cash flows for the bundle. Then, for each price, 0 is assigned if the intrinsic value at the price is less than or equal to the continuation value, and 1 is assigned otherwise, and the critical price for early exercise is chosen to be the price corresponding to a sequence of 1's which is longer than any subsequent sequences of 0's. Anderson [3] has proposed another approach belonging to this class: After simulating price paths, one proceeds backward starting from one period before expiration to choose the critical price levels which maximize the average present values of all future cash values.

The second class attempts to find the upward and downward biased priceestimators and find confidence intervals which shrink to a point estimate asymptotically as the number of simulation increases in an appropriate way. For example, Broadie and Glasserman [12] have considered a random tree and obtained a high estimate by a traditional backward induction method and a low estimate by a similar backward induction but using a branch among all the branches emanating from a state-time in the tree for the continuation value and the other branches for the exercise decision. The upward bias comes from the fact that information about future, which is not available to the option holder, is used in the backward induction, and the downward bias from that a suboptimal exercise-time is used for the simulated value. 
The third class, which is currently most popular, tries to approximate the continuation value at each time of a simulation path by regression. Tsitsiklis and Van Roy [75] and Longstaff and Schwartz [55] have proposed to use a set of basis functions to approximate the continuation value; at each point of time on each simulation path the discounted future value is regressed against basis functions to obtain an approximate analytic form, and the exercise decision is made by comparison of the current intrinsic value and the value of the approximate analytic form of the continuation value. ${ }^{4}$

Tsitsiklis and Van Roy have also proposed to use basis functions which are functions not only of the state but also of time. With these basis functions one does not need the backward induction described in the previous paragraph, but needs to carry out iteration starting from guessed initial values of the regression parameters. Tsitsiklis and Van Roy have shown that the iteration converges because the operator involved in it is a contraction. They, however, have not shown practical examples based on regression on state-time basis functions.

All the methods involving the choice of an early exercise boundary (e.g., $[3,55,73])$ produce downward-biased estimates for the price of an American option, since they involve a sub-optimal choice of the exercise time. Relying on duality results developed by Davis and Karatzas [23], and Rogers [67], Andersen and Broadie [4] have obtained an upward-biased estimate of the price.

\section{New topics: model uncertainty, backward stochastic differential equations, and real options}

In this section we review topics that are developed recently, model uncertainty, backward stochastic differential equations, and real options.

If the financial market is complete, the risk-neutral probability measure is uniquely determined, and the previously mentioned approaches are applicable for pricing American options. In case the market is incomplete, however, there are infinitely many risk-neutral probability measures consistent with the current prices of existing products, and there is an ambiguity in pricing a newly introduced derivative. In derivative pricing this typically translates into the existence of infinitely many different pricing models and model uncertainty refers to this existence multiple pricing models. In order to handle this problem, risk measures and nonlinear expectations such as $g$-expectations and $G$ expectations have been introduced (see e.g., Föllmer [26] and Peng [64]). Many of risk measures as well as nonlinear expectations can be expressed as solutions to backward stochastic differential equations (BSDE's) [64].

\footnotetext{
${ }^{4}$ Tsitsiklis and Van Roy's method is essentially the same as that of Longstaff and Schwartz, but differs only in that the former use the estimated continuation value at time $t$ as the future value of the option for computing the continuation value at time $t-1$ whereas the latter use the actual cash flow at time $t$ as the future value.
} 
Pricing American options under model uncertainty is a relatively unexplored field. Riedel [66] has studied an optimal stopping problem such as that associated with optimal exercise of an American option in the face of model uncertainty, assuming that the decision maker optimizes against the worst-case scenario as modeled in Gilboa and Schmeidler [31]. He has shown that for a complex barrier option there may exist path dependences in the worst case scenario even if the uncertainty is modeled as a straightforward generalization of an i.i.d. process. A more general treatment of an American option pricing under model uncertainty will require solving BSDE's, since that will involve risk measures and/or nonlinear expectations whose implementation is generally carried out through BSDE's.

The price of an American option can also be modeled as a backward stochastic differential equation with a reflecting barrier, where the barrier is its intrinsic value. Generalizing this, a generalized American option can be defined as a contingent claim which can be switched into other $N-1$ different regimes starting from a regime by paying appropriate fixed costs. The price of a generalized American option can be expressed as a solution to a system of $N$ BSDE's with reflecting barriers. Hu and Tang [37] have obtained results concerning existence and uniqueness for such BSDE's.

Real options are real investment opportunities involving sunk costs. The sunk costs make them resemble American options, even though continuous hedging is not usually possible (see e.g., Dixit and Pindyck [25]). Real options are very widely applied to real world investment problems: R\&D evaluation, venture capital investment, oil exploration, research on alternative energy sources or desalination $^{5}$ (See e.g., Trigeorgis [74]). Real options with multiple choices can be modeled as a generalized American option we discussed above, and their values can be obtained as solutions to a system of BSDE's with reflecting barriers.

\section{Open problems and future research}

\section{Convergence}

The important contributions, the randomization method and the homotopy method lack the proof of convergence. ${ }^{6}$ Numerical examples seem to suggest the methods' convergence to the true values. However, unless a mathematical proof is given, we are not sure whether the method is valid for all parameter values.

2. Stochastic Volatility Models and Other Multi-factor Models

Stochastic volatility models have been popular to consider the effects of stochastically changing volatilities on option pricing $([40,36])$. For the case where the speed of mean reversion of volatility is very fast and the option

\footnotetext{
${ }^{5}$ Desalination refers to transforming salted water into drinking water.

${ }^{6}$ For discrete Markovian approximations such as multinomial trees, Amin and Khanna [2] have provided a convergence proof.
} 
is away from expiration Fouque, Papanicolaou, and Sircar [28] have provided a Taylor series approximation for the price of a European option; it is equal to the sum of the Black-Scholes price with constant volatility equal to the average future volatility and correction terms for the volatility and skewness of the stochastic volatility. In the same book they have extended the following extension of their method to pricing an American option: Retain the same early exercise boundary as that of the constant volatility, where the volatility is equal to the average future volatility, formulate the LCP for the American option by using the approximate operators for the European option, and solve the LCP to obtain an approximate price of an American option which they claim to be a good approximation away from the early exercise boundary.

Except for this, however, there has been only sparse research on analytic pricing of American options in an environment where volatility is changing stochastically. $^{7}$ It will be even interesting to see an analytic solution to the perpetual option pricing problem in a stochastic volatility model such as in [36]. Or it will be interesting to see an extension of the randomization approach to this case.

There is extensive literature on pricing an American option with stochastic volatility, on multiple assets, or, more generally, with multiple sources of risk based on finite difference/element methods. Such methods include projected successive overrelaxation (PSOR) methods [77, 72], penalty methods [27, 79], multigrid methods [21, 61], operator splitting methods [41, 42, 44], componentwise splitting methods [43], etc. A comprehensive survey on the growing literature is beyond the scope of the current paper and is left as a future task.

3. Analytic Solution to the Early Exercise Boundary and Option Price

The most prominent problem in American option pricing is still to discover analytic solution to the early exercise boundary and the option price. The approximations by [17] and [80] seem close to the goal. But, as our review has shown, the foremost objective of obtaining a simple analytic solution is not achieved and waits for further innovations.

4. American Options and BSDE's with Reflecting Barriers.

We have discussed the relationship between American options and BSDE's with reflecting barriers in the previous section. Research on this relationship and associated BSDE's is in its infancy. Both theoretical research (e.g., general results on existence and uniqueness of BSDE's with reflecting barriers) and numerical analysis in this direction (e.g., Monte Carlo methods for BSDE's) is one of the most promising areas in financial mathematics.

\section{Conclusion}

In this paper we have surveyed approaches to price American options. We have shown that analytic approximations, the integral representation of the early exercise boundary, the randomization method, the homotopy method,

\footnotetext{
${ }^{7}$ Exceptions are [35] and [46].
} 
the Monte Carlo method, and applications of backward stochastic differential equations have made important innovations in the area. However, as we have indicated, convergence of the best-known approximations still needs to be proved (or disproved) and innovations are necessary to deal with stochastic volatilities and multiple sources of uncertainty. Furthermore, the ultimate objective to get a simple analytic solution is yet to be achieved.

\section{References}

[1] C. Ahn, H. Choe, and K. Lee, A long time asymptotic behavior of the free boundary for an American put, Proc. Amer. Math. Soc. 137 (2009), no. 10, 3425-3436.

[2] K. Amin and A. Khanna, Convergence of American option values from discrete-to continuous-time financial models, Math. Finance 4 (1994), no. 4, 289-304.

[3] L. Andersen, A simple approach to the pricing of Bermudan swaptions in the multifactor LIBOR market model, J. Comput. Finance 3 (2000), 1-32.

[4] L. Andersen and M. Broadie, A primal-dual simulation algorithm for pricing multidimensional American options, Management Science 50 (2004), 1222-1234.

[5] G. Barone-Adesi and R. Whaley, Efficient analytic approximation of American option values, Journal of Finance 42 (1987), 301-320.

[6] A. Bensoussan, On the theory of option pricing, Acta Appl. Math. 2 (1984), no. 2, $139-158$.

[7] F. Black and M. Scholes, The pricing of options and corporate liabilities, Journal of Political Economy 81 (1973), 637-654.

[8] M. Brennan and E. Schwartz, The valuation of American put options, Journal of Finance 32 (1977), 449-462.

[9] _ Finite difference methods and jump processes arising in the pricing of contingent claims: a synthesis, Journal of Financial and Quantitative Analysis 13 (1978), 461-474.

[10] M. Broadie and J. Detemple, American option valuation: new bounds, approximations, and a comparison of existing methods, Review of Financial Studies 9 (1996), 1211-1250.

[11] Option pricing: valuation models and applications, Management Science 50 (2004), 1145-1177.

[12] M. Broadie and P. Glasserman, Pricing American-style securities using simulation, J. Econom. Dynam. Control 21 (1997), no. 8-9, 1323-1352.

[13] P. Boyle, Options: a Monte Carlo approach, Journal of Financial Economics, 4 (1977), 328-338.

[14] D. A. Bunch and H. Johnson, The American put option and its critical stock price, Journal of Finance 55 (2000), 2333-2356.

[15] S. Byun, Numerical procedures for valuing american options, Ph. D. dissertation, KAIST, 1996.

[16] S. Byun and I. Kim, Relationship between American puts and calls on futures, J. Korean Soc. Ind. Appl. Math. 4 (2000), 11-20.

[17] P. Carr, Randomization and the American put, Review of Financial Studies 81 (1998), 597-626.

[18] P. Carr and D. Faguet, Valuing Finite-Lived Options as Perpetual, Working Paper, 1996.

[19] P. Carr, R. Jarrow, and R. Myneni, Alternative characterizations of American put options, Math. Finance 2 (1992), 87-106.

[20] X. Chen and J. Chadam, A mathematical analysis for the optimal exercise boundary of American put option, SIAM J. Math. Anal. 38 (2007), 1613-1641. 
[21] N. Clarke and K. Parrot, The multigrid solution of two-factor American put options, Technical Report 96-16, (1996), Oxford Computing Laboratory, Oxford.

[22] J. Cox, S. Ross, and M. Rubinstein, Option pricing: a simplified approach, Journal of Financial Economics 7 (1979), 141-148.

[23] M. Davis and I. Karatzas, A deterministic approach to optimal stopping, with applications, in Probability, Statistics and Optimization: A Tribute to Peter Whittle, F. Kelley, ed., (1994), 455-466, Wiley.

[24] J. Detemple, American-Style Derivatives, Chapman \& Hall/CRC Financial Mathematics Series, 2006.

[25] A. Dixit and R. Pindyck, Investment Under Uncertainty, Princeton University Press, 1994.

[26] H. Föllmer, Financial uncertainty, risk measures and robust preferences, Aspects of Mathematical Finance, 3-13, Springer, Berlin, 2008.

[27] P. Forsyth and K. Vetzal, Quadratic convergence for valuing American options using a penalty method, SIAM J. Sci. Comput. 23 (2002), no. 6, 2095-2122.

[28] J. Fouque, G. Papanicolau, and K. Sircar, Derivatives in Financial Markets with Stochastic Volatility, Cambridge University Press, 2000.

[29] B. Gao, J. Huang, and M. Subrhamanyam, The valuation of American barrier options using the decomposition technique, J. Econom. Dynam. Control 24 (2000), 1783-1827.

[30] R. Geske and H. Johnson, The American put options valued analytically, Journal of Finance 39 (1984), 229-263.

[31] I. Gilboa and Schmeidler, Maxmin expected utility with non-unique prior, J. Math. Econom. 18 (1989), 141-153.

[32] P. Glasserman, Monte Carlo Methods in Financial Engineering, Applications of Mathematics (New York), 53. Stoch. Model. Appl. Probab., Springer-Verlag, New York, 2004.

[33] O. Grabbe, The pricing of call and put options on foreign exchange, Journal of International Money and Finance 2 (1983), 239-253.

[34] S. J. Grossman, The Information Role of Prices, The MIT Press, 1989.

[35] S. Guo and Q. Zhang, Closed-form solutions for perpetual American put options with regime switching, SIAM J. Appl. Math. 64 (2004), no. 6, 2034-2049.

[36] S. L. Heston, A closed-form solution for options with stochastic volatility with applications to bond and currency options, Review of Financial Studies 6 (1993), 327-343.

[37] Y. Hu and S. Tang, Multi-dimensional BSDE with oblique reflection and optimal switching, forthcoming in Probab. Theory Related Fields, 2009.

[38] J. Huang, M. Subrahmanyam, and G. Yu, Pricing and hedging of American options: a recursive integration method, Review of Financial Studies 9 (1996), 277-300.

[39] J. Hull, Options, Futures, and Other Derivatives, 7th ed., Pearson Education, 2009.

[40] J. Hull and A. White, The pricing of options on assets with stochastic volatilities, Journal of Finance 42 (1987), 281-300.

[41] S. Ikonen and J. Toivanen, Operator splitting methods for American option pricing, Appl. Math. Lett. 17 (2004), no. 7, 809-814.

[42] _ Operator splitting methods for pricing American options with stochastic volatility, Technical Report B11/2004, University of Jyväskylä, 2004.

[43] _ Componentwise splitting methods for pricing American options with stochastic volatility, Technical Report B7/2005, University of Jyväskylä, 2005.

[44] __ Efficient numerical methods for pricing American options under stochastic volatility, Technical Report B12/2005, University of Jyväskylä, 2005.

[45] S. D. Jacka, Optimal stopping and the American put, Math. Finance 1 (1991), 1-14.

[46] B. Jang and H. Koo, American Put Options with Regime-Switching Volatility, Working Paper, 2005.

[47] H. Johnson, An analytic approximation for the American put price, Journal of Financial and Quantitative Analysis 18 (1983), 141-148. 
[48] N. Ju, Pricing an American option by approximating its early exercise boundary as a multi-piece exponential function, Review of Financial Studies 11 (1998), 627-646.

[49] I. Karatzas, On the pricing of American options, Appl. Math. Optim. 17 (1988), no. 1, 37-60.

[50] I. Karatzas and S. Shreve, Methods of Mathematical Finance, New York, SpringerVerlag, 1998.

[51] I. Kim, The analytic valuation of American options, Review of Financial Studies 3 (1990), 547-572.

[52] I. Kim and S. Byun, Optimal exercise boundary in a binomial option pricing model, Journal of Financial Engineering 3 (1994), 137-158.

[53] I. Kim and B. Jang, An Alternative Numerical Approach for Valuation of American Options: A Simple Iteration Method, Working Paper, 2008.

[54] R. A. Kuske and J. B. Keller, Optimal exercise boundary for an american put option, Appl. Math. Finance 5 (1998), 107-116.

[55] A. Longstaff and E. Schwartz, Valuing American options by simulation: a simple leastsquares approach, Review of Financial Studies 14 (2001), 113-147.

[56] R. MacDonald and M. Schröder, A parity result for American options, J. Comput. Finance 1 (1998), 5-13.

[57] L. MacMillan, An analytical approximation for the Amercian put prices, Advances in Futures and Options Research 1 (1986), 119-139.

[58] H. McKean, Appendix: a free boundary problem for the heat equation arising from a problem in mathematical economics, Industrial Management Review 6 (1965), 32-39.

[59] R. Merton, The theory of rational option pricing, Bell Journal of Economics and Management Science 4 (1973), 141-183.

[60] G. Meyer and J. van der Hoek, The Evaluation of American Options with the Method of Lines, Working Paper, 1994.

[61] Oosterlee, On multigrid for linear complementary problems with applications to American-style options, Electron. Trans. Numer. Anal. 16 (2003), 165-185.

[62] D. N. Ostrove and J. Goodman, On the early exercise boundary of the American put option, SIAM J. Appl. Math. 62 (2002), 1823-1835.

[63] M. Parkinson, Option pricing: the American put, Journal of Business 50 (1977), 21-36.

[64] S. Peng, Nonlinear expectations, nonlinear evaluations and risk measures, Stochastic methods in finance, 165-253, Lecture Notes in Math., 1856, Springer, Berlin, 2004.

[65] R. Rendleman and B. Bartter, Two-state option pricing, Journal of Finance 34 (1979), 1093-1110.

[66] F. Riedel, Optimal stopping with multiple priors, Econometrica 77 (2009), no. 3, 857908.

[67] L. Rogers, Monte Carlo valuation of American options, Math. Finance 12 (2002), no. 3, 271-286

[68] P. Samuelson, Rational theory of warrant pricing, Industrial Management Review 6 (1965), 13-31.

[69] M. Schröder, Changes of numeraire for pricing futures, forwards and options, Review of Financial Studies 12 (1989), 1143-1163.

[70] E. Schwartz, The valuation of warrants: implementing a new approach, Journal of Financial Economics 4 (1977), 79-93.

[71] R. Stamicar, D. Sevcovic, and J. Chadam, The early exercise boundary for the American put near expiry: numerical approximation, Can. Appl. Math. Q. 7 (1999), no. 4, 427444 .

[72] D. Tavella and C. Randall, Pricing Financial Instruments: The Finite difference Method, John Wiley \& Sons, 2000.

[73] J. Tilley, Valuing American options in a path simulation model, Transactions of the Society of Actuaries 45 (1993), 83-104. 
[74] L. Trigeorgis, Real Options in Capital Investment, Praeger, 1995.

[75] J. Tsitsiklis and B. Van Roy, Regression analysis for pricing complex American-style options, IEEE Transactions on Neural Networks 12 (2001), 694-703.

[76] P. van Moerbeke, On optimal stopping and free boundary problems, Arch. Ration. Mech. Anal. 60 (1975/76), no. 2, 101-148.

[77] P. Wilmott, J. Dewynne, and S. Howison, Option Pricing, Oxford University Press, 1993.

[78] L. Wu and Y. Kwok, A front-fixing finite difference method for the valuation of American options, Journal of Financial Engineering 6 (1997), 83-97.

[79] R. Zvan, P. Forsyth, and K. Vetzal, Penalty methods for American options with stochastic volatility, J. Comput. Appl. Math. 91 (1998), no. 2, 199-218.

[80] S. Zhu, An exact and explicit solution for the valuation of American put options, Quant. Finance 6 (2006), no. 3, 229-242.

Se Ryoong Ahn

Department of Business Administration

AJOU UNIVERSITY

SUWON 443-749, KorEA

E-mail address: srahn@ajou.ac.kr

HYEONG-OHK BAE

Department of Financial Engineering \& Department of Mathematics

AJOu UnIVERsity

SUWON 443-749, KoreA

E-mail address: hobae@ajou.ac.kr

Hyeng Keun KoO

Department of Financial Engineering

AJOU UNIVERSITY

Suwon 443-749, KoreA

E-mail address: hkoo@ajou.ac.kr

KIJUNG LEE

Department of Mathematics

AJOU UNIVERSITY

Suwon 443-749, KoreA

E-mail address: kijung@ajou.ac.kr 